\title{
GLOBAL PHASE PORTRAITS OF KUKLES POLYNOMIAL DIFFERENTIAL SYSTEMS WITH HOMOGENOUS POLYNOMIAL NONLINEARITIES OF DEGREE 5 HAVING A CENTER
}

\author{
JAUME LLIBRE ${ }^{1}$ AND MAURÍCIO FRONZA DA SILVA ${ }^{2}$
}

\begin{abstract}
We provide the 22 different global phase portraits in the Poincaré disk of all centers of the so called Kukles polynomial differential systems of the form $\dot{x}=-y, \dot{y}=x+Q_{5}(x, y)$, where $Q_{5}$ is a real homogeneous polynomial of degree 5 defined in $\mathbb{R}^{2}$.
\end{abstract}

\section{IntroduCtion AND STATEMENT OF THE MAIN RESUlt}

Consider a system of the form

$$
\begin{aligned}
& \dot{x}=-y, \\
& \dot{y}=x+Q_{n}(x, y),
\end{aligned}
$$

where the dot denotes the derivative with respect to the independent variable $t$ and $Q_{n}(x, y)$ is a real homogeneous polynomial of degree $n$. A system of this form was called a Kukles polynomial differential system by Giné in [4]. In [11] Volokitin and Ivanov established the following open question about these differential systems:

Open Question. Is it true that a differential system of the form (1) with nonlinearities a polynomial $Q_{n}(x, y)$ of degree higher than 2 , has a center at the origin if, and only if, its vector field is symmetric with respect to one of the coordinate axes?

For degrees $n=2,3$ the authors of the open problem already known that the answer was positive. A positive answer of this question was proved in [4] for the degrees $n=4,5$. In [5] Giné, Llibre and Valls proved that this conjecture holds for $n \geq 5$ odd, and the same authors in [6] proved the conjecture for $n \geq 6$ even.

More precisely, in [5] the authors proved that for a planar differential system of the form (1), the origin is a center if, and only if, its vector field is symmetric with respect to one of the coordinate axes.

2010 Mathematics Subject Classification. Primary 34C05.

Key words and phrases. centers, Kukles, polynomial vector fields, phase portrait, Poincaré disc. 
In this work we use this information to classify topologically the global phase portraits of the differential systems

$$
\begin{aligned}
& \dot{x}=-y, \\
& \dot{y}=x+a x^{5}+b x^{3} y^{2}+c x y^{4},
\end{aligned}
$$

in the Poincaré disk, where $a, b, c$ are real parameters. Note that systems (2) are invariant under the change of coordinates $(t, x, y) \mapsto(-t,-x, y)$. That is, the phase portrait is symmetric with respect to $y$-axis.

In $[1,8,12,13,14]$ were classified the global phase portraits of linear systems with homogeneous nonlinearities of degree 3, so in particular the phase portraits of the Kukles systems (2) of degree 3. Moreover, in [2] and [7] have been classified the global phase portraits of the Kukles systems (2) of degree 4.

Our main result is the following one.

Theorem 1. The Poincaré compactification of a system (2) is topologically equivalent to the Poincaré compactification of one of the following systems:

(i) $\dot{x}=-y, \dot{y}=x$,

(ii) $\dot{x}=-y, \dot{y}=x+A x y^{4}$,

(iii) $\dot{x}=-y, \dot{y}=x+A x^{3} y^{2}$,

(iv) $\dot{x}=-y, \dot{y}=x+A x^{3} y^{2}+C x y^{4}$,

(v) $\dot{x}=-y, \dot{y}=x+A x^{5}$,

(vi) $\dot{x}=-y, \dot{y}=x+A x^{5}+C x y^{4}$,

(vii) $\dot{x}=-y, \dot{y}=x+A x^{5}+B x^{3} y^{2}$,

(viii) $\dot{x}=-y, \dot{y}=x+A x^{5}+B x^{3} y^{2}+C x y^{4}$,

for an appropriate choice of $A \in\{-1,1\}, B, C \in \mathbb{R} \backslash\{0\}$. The phase portraits in the Poincaré disk of systems (i)-(viii) are topologically equivalent to one of the 22 phase portraits presented in Figure 1. The possibilities for the phase portraits of systems (2) and the corresponding systems (i)-(viii) are provided in Table 1. With the Program P4 we have checked that all the possibilities hold.

In the phase portraits of our figures the separatrices are thicker than orbits of the canonical regions, and thickest lines denotes lines of singularities. For more details about Program P4 see chapters 9 and 10 of [3]. We present the relation between the parameters of system (2) and the parameters of its correspondent normal form in Table 2.

This work is organized in the following way. In section 2 we introduce the basic definitions and results. Since we use the Poincaré compactification to study the orbits near the infinity, we present a summary of this technique in section 3. The Markus-Neumann-Peixoto Theorem implies that to determine the phase portrait of a given planar vector field, it is sufficient to determine the behavior of a completed separatrix skeleton, we present the ingredients of this result in section 4 . We study the finite and infinite singular points of system (2) in sections 5 and 6 , respectively. Finally, we prove Theorem 1 in section 7 . 

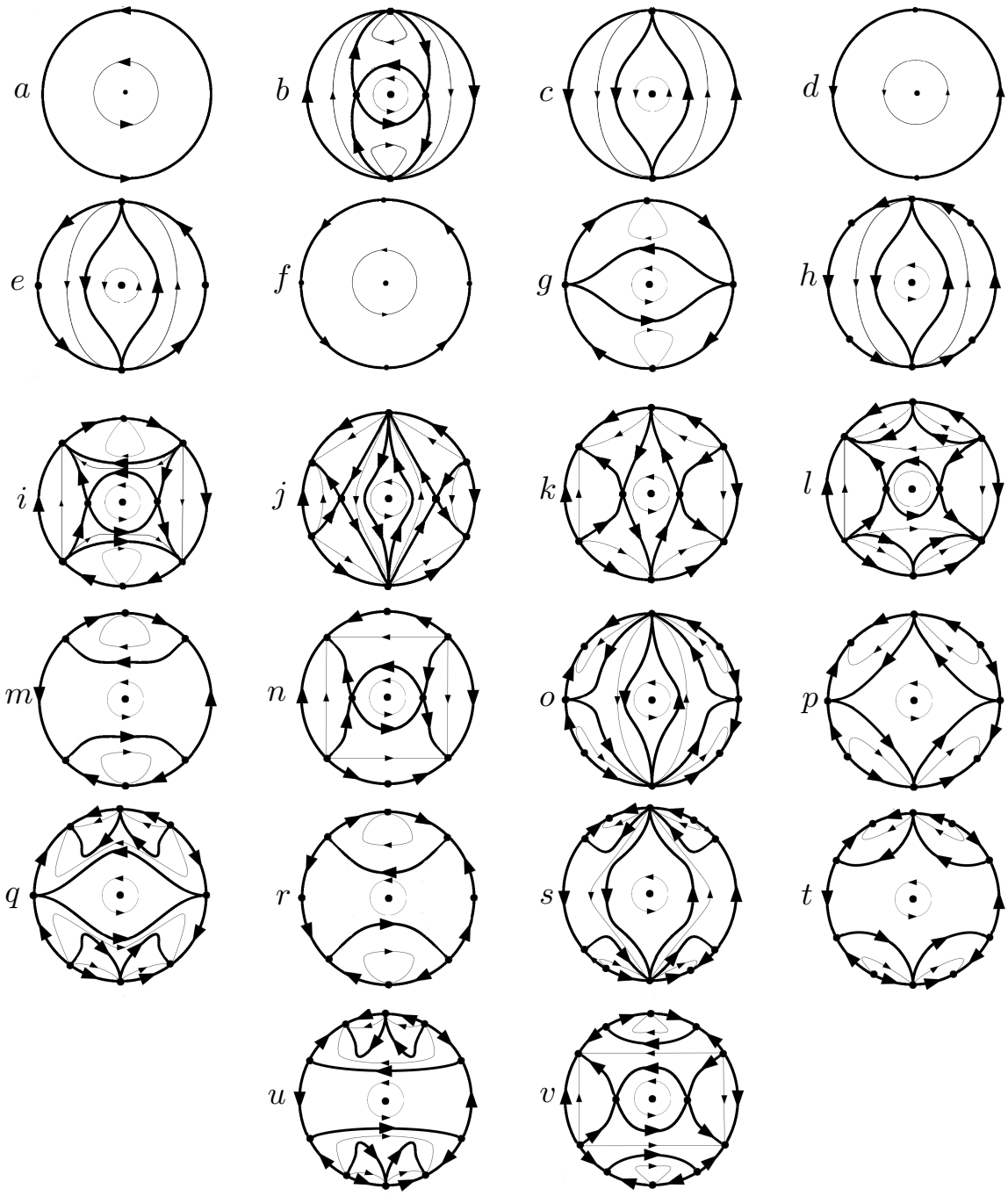

Figure 1. Phase portrait of systems (2) in the Poicaré disk.

\section{Definitions AND USEFul RESUlts}

Let $X: U \rightarrow \mathbb{R}^{2}$ be a vector field defined on the open subset of $\mathbb{R}^{2}$. Suppose that $\left(x_{0}, y_{0}\right) \in U$ is a singular point of $X$. We say that $\left(x_{0}, y_{0}\right)$ is a hyperbolic singular point of $X$ when the real part of both eigenvalues of $D X\left(x_{0}, y_{0}\right)$ are different of zero. If exactly one of the eigenvalues of $D X\left(x_{0}, y_{0}\right)$ is different of zero then $\left(x_{0}, y_{0}\right)$ is a semi-hyperbolic singular point of $X$. If $\left(x_{0}, y_{0}\right)$ is a hyperbolic or a semi-hyperbolic singular point of $X$ then is is called a elementary singularity of $X$ else, $\left(x_{0}, y_{0}\right)$ is a nonelementary singular point of $X$. 


\begin{tabular}{|c|c|c|}
\hline $\begin{array}{l}\text { Phase } \\
\text { Portrait of } \\
\text { Figure } 1\end{array}$ & System (2) & Normal Form (i)-(viii) \\
\hline $\mathrm{a}$ & $a=b=c=0$ & (i) \\
\hline $\mathrm{b}$ & $\begin{array}{l}a<0, b=c=0 \\
a<0, b>0, c<0, b^{2}-4 a c<0 \\
a<0, b<0, c<0 \\
a<0, b<0, c=0 \\
a<0, b=0, c<0\end{array}$ & $\begin{array}{l}\text { (v), } A=-1 \\
\text { (viii), } A=-1, B>0, C<0, B^{2}+4 C<0 \\
\text { (viii), } A=-1, B<0, C<0 \\
\text { (vii), } A=-1, B<0 \\
\text { (vi), } A=-1, C<0\end{array}$ \\
\hline $\mathrm{c}$ & $\begin{array}{l}a>0, b<0, c>0, b^{2}-4 a c<0 \\
a>0, b>0, c>0 \\
a>0, b=0, c>0\end{array}$ & $\begin{array}{l}\text { (viii), } A=1, B<0, C>0, B^{2}-4 C<0 \\
\text { (viii), } A=1, B>0, C>0 \\
\text { (vi), } A=1, C>0\end{array}$ \\
\hline $\mathrm{d}$ & $\begin{array}{l}a>0, b=c=0 \\
a>0, b>0, c=0\end{array}$ & $\begin{array}{l}\text { (v), } A=1 \\
\text { (vii), } A=1, B>0\end{array}$ \\
\hline e & $\begin{array}{l}a=b=0, c>0 \\
a=0, b>0, c>0\end{array}$ & $\begin{array}{l}\text { (ii), } A=1 \\
\text { (iv), } A=1, C>0\end{array}$ \\
\hline $\mathrm{f}$ & $a=0, b>0, c=0$ & (iii), $A=1$ \\
\hline $\mathrm{g}$ & $\begin{array}{l}a=b=0, c<0 \\
a=0, b<0, c=0 \\
a=0, b<0, c<0\end{array}$ & $\begin{array}{l}\text { (ii), } A=-1 \\
\text { (iii), } A=-1 \\
\text { (iv), } A=-1, C<0\end{array}$ \\
\hline $\mathrm{h}$ & $a>0, b<0, c>0, b^{2}-4 a c=0$ & (viii), $A=1, B<0, C>0, B^{2}-4 C=0$ \\
\hline i & $a<0, b>0, c<0, b^{2}-4 a c=0$ & (viii), $A=-1, B>0, C<0, B^{2}+4 C=0$ \\
\hline $\mathrm{j}, \mathrm{k}, \mathrm{l}$ & $\begin{array}{l}a<0, b>0, c>0 \\
a<0, b<0, c>0 \\
a<0, b=0, c>0\end{array}$ & $\begin{array}{l}\text { (viii), } A=-1, B>0, C>0 \\
\text { (viii), } A=-1, B<0, C>0 \\
\text { (vi), } A=-1, C>0\end{array}$ \\
\hline $\mathrm{m}$ & $\begin{array}{l}a>0, b \neq 0, c<0 \\
a>0, b<0, c=0 \\
a>0, b=0, c<0\end{array}$ & $\begin{array}{l}\text { (viii), } A=1, C<0 \\
\text { (vii), } A=1, B<0 \\
\text { (vi), } A=1, C<0\end{array}$ \\
\hline $\mathrm{n}$ & $a<0, b>0, c=0$ & (vii), $A=-1, B>0$ \\
\hline $\mathrm{o}, \mathrm{p}, \mathrm{q}$ & $a=0, b<0, c>0$ & (iv), $A=-1, C>0$ \\
\hline $\mathrm{r}$ & $a=0, b>0, c<0$ & (iv), $A=1, C<0$ \\
\hline $\mathrm{s}, \mathrm{t}, \mathrm{u}$ & $a>0, b<0, c>0, b^{2}-4 a c>0$ & (viii), $A=1, B<0, C>0, B^{2}-4 C>0$ \\
\hline $\mathrm{v}$ & $a<0, b>0, c<0, b^{2}-4 a c>0$ & (viii), $A=-1, B>0, C<0, B^{2}+4 C>0$ \\
\hline
\end{tabular}

TABLE 1. Classification of phase portraits and normal forms of systems (2).

Here we use the definitions of (stable and unstable) node, saddle point, elliptic, hyperbolic and parabolic sector (attracting or repelling) as in [3].

In this work, the topological behavior of the flow near a hyperbolic singular point of $X$ is given by Theorem 2.15 of [3]. To determine the behavior of the flow near a semi-hyperbolic singular point we use Theorem 2.19 of [3]. 
If a non-elementary singular point $\left(x_{0}, y_{0}\right)$ of $X$ is such that both the eigenvalues of $D X\left(x_{0}, y_{0}\right)$ are zero, but $D X\left(x_{0}, y_{0}\right)$ is not zero, then $\left(x_{0}, y_{0}\right)$ is called nilpotent singularity. If $D X\left(x_{0}, y_{0}\right)$ is the null matrix then $\left(x_{0}, y_{0}\right)$ is a linearly zero singularity. The study of nilpotent singular points is performed by the change of coordinates of the form $x \mapsto x, y \mapsto x y$, and $x \mapsto x y, y \mapsto y$, called homogeneous blow-up or, simply blow-up, and it is summarized in Theorem 3.5 of [3].

But, all the nonelementary singularities of system (2) are linearly zero. To study these singular points we will use quasihomogeneous blow-up, that is, a change of variables of the form

$$
\begin{aligned}
& (x, y) \mapsto\left(x^{\alpha}, x^{\beta} y\right), \text { positive } x \text {-direction, } \\
& (x, y) \mapsto\left(-x^{\alpha}, x^{\beta} y\right), \text { negative } x \text {-direction, } \\
& (x, y) \mapsto\left(x y^{\alpha}, y^{\beta}\right), \text { positive } y \text {-direction, } \\
& (x, y) \mapsto\left(x y^{\alpha},-y^{\beta}\right), \text { negative } y \text {-direction, }
\end{aligned}
$$

where $\alpha, \beta$ are positive integers. For quasihomogeneous blow-ups in the $x$-direction (respectively $y$-direction), when $\alpha$ (respectively $\beta$ ) is odd, the information obtained in the positive $x$-direction (respectively $y$-direction) is useful to the negative $x$-direction (respectively $y$-direction). To avoid successive blow-up's we choose $\alpha$ and $\beta$ using the Newton diagram (see p. 104 of [3]).

\section{Poincaré compactification}

In this section we present a technique useful to study the behavior of the trajectories of polynomial vector fields near the infinity. More details in p. 149 of [3].

Consider the polynomial vector field $X$ defined on $\mathbb{R}^{2}$ by system

$$
\begin{aligned}
& \dot{x}=P(x, y), \\
& \dot{y}=Q(x, y),
\end{aligned}
$$

where $P$ and $Q$ are real polynomial functions defined on $\mathbb{R}^{2}$. We define the degree $d$ of $X$ as the maximum between the degree of $P$ and $Q$.

We consider the notation $\mathbb{S}^{2}=\left\{\left(z_{1}, z_{2}, z_{3}\right) \in \mathbb{R}^{3} ; z_{1}^{2}+z_{2}^{2}+z_{3}^{2}=1\right\}, \mathbb{S}^{1}=$ $\left\{\left(z_{1}, z_{2}, z_{3}\right) \in \mathbb{S}^{2} ; z_{3}=0\right\}$ and identify $\mathbb{R}^{2}$ with the tangent plane $\pi$ of $\mathbb{S}^{2}$ at the north pole $(0,0,1)$. If we consider the central projection of $\pi$ in $\mathbb{S}^{2}$, we obtain a tangent vector field defined on $\mathbb{S}^{2} \backslash \mathbb{S}^{1}$ such that the infinity points of $\pi$ are projected in $\mathbb{S}^{1}$.

This vector field is symmetric about the center of $\mathbb{S}^{2}$ and, in general, is unbounded near $\mathbb{S}^{1}$. After a multiplication by an appropriate factor, the resultant vector field admits an analytical extension to $\mathbb{S}^{2}$. Due to the symmetry, it is sufficient to consider the extended vector field defined only in the closed northern hemisphere $H$ of $\mathbb{S}^{2}$. The ortogonal projection of $H$ into the disk $\left\{\left(z_{1}, z_{2}, z_{3}\right) \in \mathbb{R}^{3} ; z_{1}^{2}+z_{2}^{2} \leq 1, z_{3}=0\right\}$ is called the Poincaré disk. 
More precisely, we denote the northern and southern hemisphere of $\mathbb{S}^{2}$ by $H^{+}=\left\{\left(z_{1}, z_{2}, z_{3}\right) \in \mathbb{S}^{2} ; z_{3}>0\right\}$ and $H^{-}=\left\{\left(z_{1}, z_{2}, z_{3}\right) \in \mathbb{S}^{2} ; z_{3}<0\right\}$, respectively. The central projections of $\pi:=\left\{\left(z_{1}, z_{2}, z_{3}\right) \in \mathbb{R}^{3} ; z_{3}=1\right\}$ in $H^{+}$and $H^{-}$are defined, respectively, by

$$
\begin{aligned}
f^{+}: \pi & \rightarrow H^{+} & f^{-}: \pi & \rightarrow H^{-} \\
z & \mapsto \frac{1}{\Delta(z)}\left(z_{1}, z_{2}, 1\right), & z & \mapsto \frac{1}{\Delta(z)}\left(-z_{1},-z_{2},-1\right),
\end{aligned}
$$

where $\Delta(z)=\sqrt{z_{1}^{2}+z_{2}^{2}+1}, z \in \pi$. Define the vector field $\bar{X}$ on $\mathbb{S}^{2} \backslash \mathbb{S}^{1}$ by

$$
\bar{X}(w)=\left\{\begin{array}{l}
D f^{+}(z) X(z), \text { if } w=f^{+}(z), \\
D f^{-}(z) X(z), \text { if } w=f^{-}(z) .
\end{array}\right.
$$

In general $\bar{X}$ is unbounded in a neighborhood of $\mathbb{S}^{1}$, then there is no extension of $\bar{X}$ to $\mathbb{S}^{2}$. But we can prove that if we multiply $\bar{X}$ by $w_{3}^{d}$ then the resultant vector field has an unique analytical extension to $\mathbb{S}^{2}$, called the Poincaré compactification of $X$ and denoted by $p(X)$.

Notice that, in general, in each hemisphere we have that $p(X)$ is $C^{\omega}$ equivalent, but not $C^{\omega}$-conjugated, to $X$. Then we can study the behavior of the trajectories of $X$ near a singularity using the correspondent singularities of $p(X)$. But it may be that $p(X)$ has singularities in $\mathbb{S}^{1}$. A singular point of $p(X)$ which belongs to $\mathbb{S}^{2} \backslash \mathbb{S}^{1}$ (respectively $\mathbb{S}^{1}$ ) is called finite (respectively infinite) singular point of $X$. We can prove that $\mathbb{S}^{1}$ is invariant under the flow of $p(X)$.

From the definition of $f^{+}$and $f^{-}$it follows that $p(X)$ is symmetric with respect to the origin, then to study the trajectories of $X$ it is sufficient to study $p(X)$ in the closed northern hemisphere $H^{+} \cup \mathbb{S}^{1}$.

To obtain expressions of $p(X)$ in local coordinates, we consider the charts of the sphere $\mathbb{S}^{2}$. For $j=1,2,3$ define $U_{j}=\left\{\left(z_{1}, z_{2}, z_{3}\right) \in \mathbb{S}^{2} ; z_{j}>0\right\}$, $V_{j}=\left\{\left(z_{1}, z_{2}, z_{3}\right) \in \mathbb{S}^{2} ; z_{j}<0\right\}$ and $\varphi_{j}: U_{j} \rightarrow \mathbb{R}^{2}, \psi_{j}: V_{j} \rightarrow \mathbb{R}^{2}$ given by

$$
\varphi_{1}(z)=-\psi_{1}(z)=\frac{\left(z_{2}, z_{3}\right)}{z_{1}}, \varphi_{2}(z)=-\psi_{2}(z)=\frac{\left(z_{1}, z_{3}\right)}{z_{2}}, \varphi_{3}(z)=\frac{\left(z_{1}, z_{2}\right)}{z_{3}} .
$$

If we denote by $(u, v)$ the value of $\varphi_{j}$ or $\psi_{j}$ at the point $z$ we can prove that the expression of $p(X)$ in the chart $\left(U_{1}, \varphi_{1}\right)$ is given by

$$
\begin{aligned}
\dot{u} & =v^{d}\left[-u P\left(\frac{1}{v}, \frac{u}{v}\right)+Q\left(\frac{1}{v}, \frac{u}{v}\right)\right], \\
\dot{v} & =-v^{d+1} P\left(\frac{1}{v}, \frac{u}{v}\right) .
\end{aligned}
$$

The expression of $p(X)$ in the chart $\left(U_{2}, \varphi_{2}\right)$ is

$$
\begin{aligned}
\dot{u} & =v^{d}\left[P\left(\frac{u}{v}, \frac{1}{v}\right)-u Q\left(\frac{u}{v}, \frac{1}{v}\right)\right], \\
\dot{v} & =-v^{d+1} Q\left(\frac{u}{v}, \frac{1}{v}\right),
\end{aligned}
$$


and the expression of $p(X)$ in the chart $\left(U_{3}, \varphi_{2}\right)$ is

$$
\begin{aligned}
& \dot{u}=P(u, v), \\
& \dot{v}=Q(u, v) .
\end{aligned}
$$

Finally, for each $j=1,2,3$, the expression of $p(X)$ in the chart $\left(V_{j}, \psi_{j}\right)$ is the expression of $p(X)$ in the chart $\left(U_{j}, \varphi_{j}\right)$ multiplied by the factor $(-1)^{d-1}$.

Using this notation we observe that if $(u, v) \in U_{j}$ is a infinite singular point of $X$ if, and only if, the expression of $p(X)$ in the chart $\left(U_{j}, \varphi_{j}\right)$ vanishes in $(u, v)$ and $v=0$.

Notice that if $z$ is a infinite singular point of $X$ then $-z$ is also a infinite singular point of $X$. In this case, from the expressions of $p(X)$ in local coordinates it follows that the behavior of the flow near $-z$ can be determined by the behavior of the flow near $z$, because the flow near $-z$ differs by the flow near $z$ by the factor $(-1)^{d-1}$. Then the study of $p(X)$ in the charts $\left(V_{j}, \psi_{j}\right), j=1,2,3$, is superfluous.

Moreover, notice that if $z$ is a infinite singular point of $X$ with $z \in U_{2}, z \neq$ $(0,1,0)$ then $z \in U_{1} \cup V_{1}$. It follows that to study all the infinite singular points of $X$, it is sufficient to study the singularities of $p(X)$ in $U_{1}$ and the origin of $U_{2}$

\section{Markus-Neumann-Peixoto Theorem}

In this section we present the Markus-Neumann-Peixoto Theorem, which reduce the work of determine the phase portrait of a given planar vector fields, to the determination of the separatrices (see definition below) and a finite number of special orbits. More details in [9], [10] or p. 33 of [3]. It is sufficient to consider $C^{1}$-vector fields.

Let $X$ an $Y$ be $C^{1}$-vector fields defined on the open sets $U$ and $V$ of $\mathbb{R}^{2}$, respectively. Using the notation of Markus and Neumann, we denote by $(U, \Phi)$ and $(V, \Psi)$ the flow of $X$ and $Y$, respectively. We say that $(U, \Phi)$ and $(V, \Psi)$ are topologically equivalent if there exists a homeomorphism of $U$ in $V$ which carries the orbits of $X$ in orbits of $Y$, preserving the orientation of the all orbits. If $(U, \Phi)$ and $(V, \Psi)$ are topologically equivalent then we also say that their phase portraits are topologically equivalent.

Suppose that $U=\mathbb{R}^{2}$. We say that the flow $\left(\mathbb{R}^{2}, \Phi\right)$ is parallel if it is topologically equivalent of the flow $(V, \Psi)$ define by one of the following vector fields:

- $Y$ defined on $\mathbb{R}^{2}$ by $Y(x, y)=(1,0), \forall(x, y) \in \mathbb{R}^{2}$,

- $Y$ defined on $\mathbb{R}^{2} \backslash\{(0,0)\}$ such that, in polar coordinates, $Y$ is given by $\dot{r}=0, \dot{\theta}=1$,

- $Y$ defined on $\mathbb{R}^{2} \backslash\{(0,0)\}$ such that, in polar coordinates, $Y$ is given by $\dot{r}=r, \dot{\theta}=0$.

The flow of the above three vector fields are called strip flow, annulus flow and nodal flow, respectively. 
Given $p \in U$, we denote the orbit, the $\alpha$-limit and the $\omega$-limit of $p$ the by $\gamma(p), \alpha(p)$ and $\omega(p)$, respectively. We say that $\gamma(p)$ is a separatrix if

- $\gamma(p)$ is a singular point, or

- $\gamma(p)$ is a periodic orbit and there is no neighborhood of $\gamma(p)$ consisting of periodic orbits, or

- $\gamma(p)$ is homeomorphic to $\mathbb{R}$ and there is no neighborhood $W$ of $\gamma(p)$ with the following two properties:

$-q \in W \Rightarrow \alpha(q)=\alpha(p)$ and $\omega(q)=\omega(p)$,

- the boundary of $W$ is composed by $\alpha(p), \omega(p)$ and by two another orbits $\gamma\left(p_{1}\right), \gamma\left(p_{2}\right)$ such that $\alpha\left(p_{1}\right)=\alpha\left(p_{2}\right)=\alpha(p)$ and $\omega\left(p_{1}\right)=\omega\left(p_{2}\right)=\omega(p)$.

The union of all separatrices of a given flow $(U, \Phi)$ is called extended separatrix skeleton, which is a closed invariant subset of $U$ and denoted by $\Sigma$. Each connected component of $U \backslash \Sigma$ is an open invariant set, called a canonical region. The next result means that there is only three possibilities for the flow in each canonical region.

Proposition 2. In each canonical region, the flow is parallel.

The completed separatrix skeleton is the union of the extended separatrix skeleton with one orbit in each canonical region. Given the flows $\left(\mathbb{R}^{2}, \Phi\right)$ and $\left(\mathbb{R}^{2}, \Psi\right)$, consider its extended separatrix skeleton $C_{1}$ and $C_{2}$, respectively. We say that $C_{1}$ and $C_{2}$ are topologically equivalent if there exist a homeomorphism of $\mathbb{R}^{2}$ in $\mathbb{R}^{2}$ which map orbits of $C_{1}$ in orbits of $C_{2}$ preserving the orientation.

Finally we present the Markus-Newmann-Peixoto theorem. This result implies that, to draw the phase portrait of a given planar vector field, it is sufficient determine its separatrices and the behavior of one orbit in each canonical region.

Theorem 3 (Markus-Neumann-Peixoto). Suppose that $\left(\mathbb{R}^{2}, \Phi\right)$ and $\left(\mathbb{R}^{2}, \Psi\right)$ are continuous flows with only isolated singular points. Then $\left(\mathbb{R}^{2}, \Phi\right)$ and $\left(\mathbb{R}^{2}, \Psi\right)$ are topologically equivalent if, and only if, its completed separatrices skeleton are topologically equivalent.

\section{Symmetries and finite Singular Points of System (2)}

From now on $X$ is the vector field defined by system (2). Additionally to the symmetry of $X$ with respect to the $y$-axis, $X$ is invariant under the change of coordinates $(x, y) \mapsto(-x,-y)$. Then, to draw the phase portrait of $X$ it is sufficient to study the quadrant given by $x, y \geq 0$ in the Poincaré disk.

We study the finite singular points of system (2) using Theorem 2.15 of [3] in the following remark. 
Remark 4. If $a \geq 0$ then $(0,0)$ is the unique singularity of (2) and, if $a<0$ then there exist another two singularities, $( \pm 1 / \sqrt[4]{-a}, 0)$. In this case

$$
D X\left( \pm \frac{1}{\sqrt[4]{-a}}, 0\right)=\left(\begin{array}{cc}
0 & -1 \\
-4 & 0
\end{array}\right)
$$

and there are hyperbolic saddle points. Moreover, $[1,-2]$ and $[1,2]$ are eigenvectors associated to the eigenvalues 2 and -2 , respectively.

\section{INFINITE SINGULAR POINTS OF SYSTEM}

To study the infinity singularities of $X$, we observe that the Poincaré compactification of $X$ in the charts $\left(U_{1}, \varphi_{1}\right)$ and $\left(U_{2}, \varphi_{2}\right)$ is given by

$$
\begin{aligned}
& \dot{u}=\left(1+u^{2}\right) v^{4}+p(u), \\
& \dot{v}=u v^{5},
\end{aligned}
$$

and

$$
\begin{aligned}
& \dot{u}=-\left(1+u^{2}\right) v^{4}-u^{2} q(u) \\
& \dot{v}=-u v^{5}-u v q(u)
\end{aligned}
$$

respectively, where

$$
p(u)=c u^{4}+b u^{2}+a \text { and } q(u)=a u^{4}+b u^{2}+c .
$$

We will perform the study of the infinite singular points in $U_{1}$ and the origin of $U_{2}$ in the subsections 6.1 and 6.2 , respectively.

6.1. Infinity singular points in $U_{1}$. In this section we study the local phase portrait of the infinite singular points of (2) which belong to $U_{1}$. Note that

Remark 5. $\left(u_{0}, 0\right)$ is a singular point of (3) if, and only if, $p\left(u_{0}\right)=0$.

Suppose that $\left(u_{0}, 0\right)$ is a singularity of system $(3)$. From the both symmetries of system (3) it follows that it is sufficient to study the infinity singular points in $U_{1}$ of the form $\left(u_{0}, 0\right)$ with $u_{0} \geq 0$.

The Jacobian matrix of the vector field defined by system (3) evaluated at $\left(u_{0}, 0\right)$ is

$$
\left(\begin{array}{cc}
p^{\prime}\left(u_{0}\right) & 0 \\
0 & 0
\end{array}\right)
$$

then $\left(u_{0}, 0\right)$ is a semi-hyperbolic or a linearly zero singular point of (3).

6.1.1. Semi-hyperbolic singular points. The semi-hyperbolic singular points in $U_{1}$ are treated in the next result.

Lemma 6. Suppose that $p\left(u_{0}\right)=0$ and $p^{\prime}\left(u_{0}\right) \neq 0$. Then $u_{0} \neq 0$, moreover if $u_{0}>0$ and $p^{\prime}\left(u_{0}\right)>0$ then $\left(u_{0}, 0\right)$ is a semi-hyperbolic unstable node of system (3). If $u_{0}>0$ and $p^{\prime}\left(u_{0}\right)<0$ then $\left(u_{0}, 0\right)$ is a semi-hyperbolic saddle point of system (3). 
Proof. From the definition of $p$ it follows that $u_{0} \neq 0$. After the translation $u \mapsto u-u_{0}$ system (3) becomes

$$
\begin{aligned}
& \dot{u}=\left(1+\left(u+u_{0}\right)^{2}\right) v^{4}+p\left(u+u_{0}\right), \\
& \dot{v}=\left(u+u_{0}\right) v^{5} .
\end{aligned}
$$

Define

$$
\begin{aligned}
& A(u, v)=\left(1+\left(u+u_{0}\right)^{2}\right) v^{4}+p\left(u+u_{0}\right)-p^{\prime}\left(u_{0}\right) u, \\
& B(u, v)=\left(u+u_{0}\right) v^{5},
\end{aligned}
$$

then we can write system (6) of the form

$$
\begin{aligned}
& \dot{u}=p^{\prime}\left(u_{0}\right) u+A(u, v), \\
& \dot{v}=B(u, v) .
\end{aligned}
$$

Let $f=f(v)$ be the solution of the equation $p^{\prime}\left(u_{0}\right) f(v)+A(f(v), v)=0$ in a neighborhood of $(0,0)$ and define $g(v)=B(f(v), v)$. Then

$$
g^{(j)}(0)=0, j=0,1,2,3,4, g^{(5)}(0)=5 ! u_{0} .
$$

From Theorem 2.19 of [3] it follows Lemma 6.

6.1.2. Nonelementary singular points. Suppose that $p\left(u_{0}\right)=p^{\prime}\left(u_{0}\right)=0$, then $\left(u_{0}, 0\right)$ is a linearly zero singular point of system $(3)$ and we use quasihomogeneous blow-up to study this singular point. The choice of the exponents $\alpha, \beta$ of the quasihomogeneous blow-up is given by the Newton diagram and depends of the order of vanishing of $p$ at $u_{0}$.

Lemma 7. Assume that $p\left(u_{0}\right)=p^{\prime}\left(u_{0}\right)=0$. Then we can write $p$ of the form $p(u)=\left(u-u_{0}\right)^{N} \widetilde{p}\left(u-u_{0}\right)$, for a suitable choice of $N=2$ or $N=4$. Moreover,

$$
N=2 \Rightarrow \widetilde{p}(u)=c u^{2}+4 c u_{0} u+6 c u_{0}^{2}+b, \widetilde{p}(0)=\frac{p^{\prime \prime}\left(u_{0}\right)}{2},
$$

and case $N=4$ corresponds to $a=b=0, c \neq 0, \widetilde{p} \equiv c$.

Proof. It is sufficient to show that the case $N=3$ is impossible. It is true when $c=0$. Suppose that $c \neq 0$ and $p^{\prime \prime \prime}\left(u_{0}\right)=24 c u_{0} \neq 0$, then $u_{0} \neq 0$. Since $p$ is an even function $p\left(-u_{0}\right)=0$. In the other hand, if we take $u=-u_{0}$ on the Taylor's formula $p(u)=\left(u-u_{0}\right)^{3}\left(4 c u_{0}+c\left(u-u_{0}\right)\right)$, we obtain $p\left(-u_{0}\right)=-16 c u_{0}^{4} \neq 0$.

In Lemmas 8 and 9 we consider the cases $N=2$ and $N=4$, respectively.

Lemma 8. Suppose that $p\left(u_{0}\right)=p^{\prime}\left(u_{0}\right)=0$. If $u_{0} \geq 0$ and $p^{\prime \prime}\left(u_{0}\right)>0$ then the sectorial decomposition of system $(3)$ at $\left(u_{0}, 0\right)$ is composed by two hyperbolic sectors (Figure 3 ). If $u_{0}=0$ and $p^{\prime \prime}(0)<0$ then the sectorial decomposition of system $(3)$ at $\left(u_{0}, 0\right)$ is composed by six hyperbolic sectors (Figure 5). If $u_{0}>0$ and $p^{\prime \prime}\left(u_{0}\right)<0$ then the sectorial decomposition of system $(3)$ at $\left(u_{0}, 0\right)$ is composed by two hyperbolic sectors and one parabolic repelling sector, that is, it is a saddle-node (Figure 7). 
Proof. Suppose that $u_{0} \geq 0$. After the translation $u \mapsto u-u_{0}$ and using notation of Lemma 7, system (3) becomes

$$
\begin{aligned}
& \dot{u}=\left(1+\left(u+u_{0}\right)^{2}\right) v^{4}+u^{2} \widetilde{p}(u), \\
& \dot{v}=\left(u+u_{0}\right) v^{5} .
\end{aligned}
$$

Notice that for system (9) we have

$$
u=0, v \neq 0 \Rightarrow \dot{u}>0 .
$$

We take the quasihomogeneous blow-up in the positive $u$-direction defined by $u=\bar{u}^{2}, v=\bar{u} \bar{v}$, which carries system (9) into

$$
\begin{aligned}
& \dot{\bar{u}}=\frac{1}{2} \bar{u}^{3}\left[\left(1+\left(\bar{u}^{2}+u_{0}\right)^{2}\right) \bar{v}^{4}+\widetilde{p}\left(\bar{u}^{2}\right)\right], \\
& \dot{\bar{v}}=\left(\bar{u}^{2}+u_{0}\right) \bar{u}^{4} \bar{v}^{5}-\frac{1}{2} \bar{u}^{2} \bar{v}\left[\left(1+\left(\bar{u}^{2}+u_{0}\right)^{2}\right) \bar{v}^{4}+\widetilde{p}\left(\bar{u}^{2}\right)\right] .
\end{aligned}
$$

Doing the rescaling of the independent variable given by $d t=\bar{u}^{2} d s$, system

(11) becomes

$$
\begin{aligned}
& \bar{u}^{\prime}=\frac{1}{2} \bar{u}\left[\left(1+\left(\bar{u}^{2}+u_{0}\right)^{2}\right) \bar{v}^{4}+\widetilde{p}\left(\bar{u}^{2}\right)\right], \\
& \bar{v}^{\prime}=\left(\bar{u}^{2}+u_{0}\right) \bar{u}^{2} \bar{v}^{5}-\frac{1}{2} \bar{v}\left[\left(1+\left(\bar{u}^{2}+u_{0}\right)^{2}\right) \bar{v}^{4}+\widetilde{p}\left(\bar{u}^{2}\right)\right],
\end{aligned}
$$

where the prime denotes derivative with respect to $s$.

The singularities of system (12) with $\bar{u}=0$ are isolated and are given by $\left(0, \bar{v}_{0}\right)$, where $\bar{v}_{0}=0$ or $\bar{v}_{0}$ is a solution of the equation

$$
\bar{v}_{0}^{4}=-\widetilde{p}(0) /\left(1+u_{0}^{2}\right) .
$$

The Jacobian matrix of the vector-field defined by system (12) evaluated at $\left(0, \bar{v}_{0}\right)$ is

$$
\left(\begin{array}{cc}
\frac{1}{2}\left[\left(1+u_{0}^{2}\right) \bar{v}_{0}^{4}+\widetilde{p}(0)\right] & 0 \\
0 & -\frac{1}{2}\left[5\left(1+u_{0}^{2}\right) \bar{v}_{0}^{4}+\widetilde{p}(0)\right]
\end{array}\right) .
$$

If $\widetilde{p}(0)>0$ then the origin is the unique singular point of system (12) with $\bar{u}=0$ and it is a hyperbolic saddle point.

If $\widetilde{p}(0)<0$, then there are three singular points of system (12) with $\bar{u}=0$, namely: $(0,0)$ which is a hyperbolic saddle point, and $\left(0, \bar{v}_{0}\right)$, with $\bar{v}_{0}$ given by the two real solutions of the equation (13), which are semi-hyperbolic saddle points.

Now, we take the quasihomogeneous blow-up in the negative $u$-direction defined by $u=-\bar{u}^{2}, v=\bar{u} \bar{v}$, which carries system (9) into

$$
\begin{aligned}
& \dot{\bar{u}}=-\frac{1}{2} \bar{u}^{3}\left[\left(1+\left(-\bar{u}^{2}+u_{0}\right)^{2}\right) \bar{v}^{4}+\widetilde{p}\left(-\bar{u}^{2}\right)\right], \\
& \dot{\bar{v}}=\left(-\bar{u}^{2}+u_{0}\right) \bar{u}^{4} \bar{v}^{5}+\frac{1}{2} \bar{u}^{2} \bar{v}\left[\left(1+\left(-\bar{u}^{2}+u_{0}\right)^{2}\right) \bar{v}^{4}+\widetilde{p}\left(-\bar{u}^{2}\right)\right] .
\end{aligned}
$$

Doing the rescaling of the independent variable given by $d t=\bar{u}^{2} d s$, system

(14) becomes

$$
\begin{aligned}
& \bar{u}^{\prime}=-\frac{1}{2} \bar{u}\left[\left(1+\left(-\bar{u}^{2}+u_{0}\right)^{2}\right) \bar{v}^{4}+\widetilde{p}\left(-\bar{u}^{2}\right)\right], \\
& \bar{v}^{\prime}=\left(-\bar{u}^{2}+u_{0}\right) \bar{u}^{2} \bar{v}^{5}+\frac{1}{2} \bar{v}\left[\left(1+\left(-\bar{u}^{2}+u_{0}\right)^{2}\right) \bar{v}^{4}+\widetilde{p}\left(-\bar{u}^{2}\right)\right] .
\end{aligned}
$$


The singular points of system (15) with $\bar{u}=0$ are isolated and given by $\left(0, \bar{v}_{0}\right)$, where $\bar{v}_{0}=0$ or $\bar{v}_{0}$ defined by the real solutions of (13). The Jacobian matrix of the vector-field defined by system (15) evaluated at $\left(0, \bar{v}_{0}\right)$ is

$$
\left(\begin{array}{cc}
-\frac{1}{2}\left[\left(1+u_{0}^{2}\right) \bar{v}_{0}^{4}+\widetilde{p}(0)\right] & 0 \\
0 & \frac{1}{2}\left[5\left(1+u_{0}^{2}\right) \bar{v}_{0}^{4}+\widetilde{p}(0)\right]
\end{array}\right) .
$$

If $\widetilde{p}(0)>0$ then the origin is the unique singular point of system (15) with $\bar{u}=0$, and it is a hyperbolic saddle point.

If $\widetilde{p}(0)<0$, then there are three singular points of system (15) with $\bar{u}=0$, namely: $(0,0)$ which is a hyperbolic saddle point, and $\left(0, \bar{v}_{0}\right)$, with $\bar{v}_{0}$ given by the two real solutions of equation (13), which are semi-hyperbolic singular points. Moreover, if $u_{0}=0$ then $\left(0, \bar{v}_{0}\right)$ is a semi-hyperbolic saddle point and if $u_{0}>0$ then $\left(0, \bar{v}_{0}\right)$ is a semi-hyperbolic unstable node.

For case $u_{0} \geq 0, p\left(u_{0}\right)=p^{\prime}\left(u_{0}\right)=0$ and $p^{\prime \prime}\left(u_{0}\right)>0$, we summarize the blow-ups in Figure 2. From (10) it follows that the sectorial decomposition of system $(3)$ at $\left(u_{0}, 0\right)$ is given in Figure 3.

For case $u_{0}=0, p(0)=p^{\prime}(0)=0$ and $p^{\prime \prime}(0)<0$, we summarize the blowups in Figure 4. From (10) it follows that the sectorial decomposition of system (3) at the origin of $U_{1}$ in Figure 5.

For case $u_{0}>0, p\left(u_{0}\right)=p^{\prime}\left(u_{0}\right)=0$ and $p^{\prime \prime}\left(u_{0}\right)<0$, we summarize the blow-ups in Figure 6. From (10) it follows that the sectorial decomposition of system $(3)$ at $\left(u_{0}, 0\right)$ is given in Figure 7.
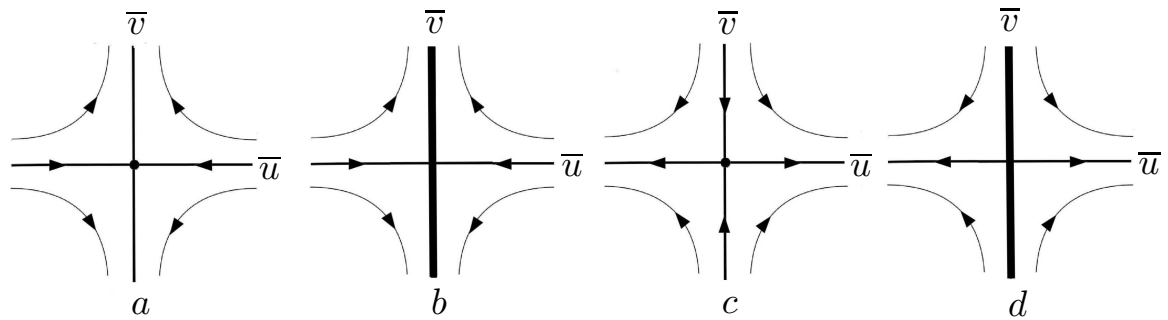

Figure 2. Case $u_{0} \geq 0, p\left(u_{0}\right)=p^{\prime}\left(u_{0}\right)=0$ and $p^{\prime \prime}\left(u_{0}\right)>0$. (a) Blow-up in the negative $u$-direction, system (15), (b) system (14), (c) blow-up in the positive $u$-direction, system (12), (d) system (11).

Lemma 9. If $a=b=0$ and $c>0$ then the sectorial decomposition of system (3) at the origin is composed by two hyperbolic sectors (Figure 8-c). If $a=b=0$ and $c<0$ then the sectorial decomposition of system (3) at the origin is composed by six hyperbolic sectors (Figure 10).

Proof. Suppose that $a=b=0$ and $c \neq 0$. In this case for system (3) we have

$$
u=0, v \neq 0 \Rightarrow \dot{u}>0
$$




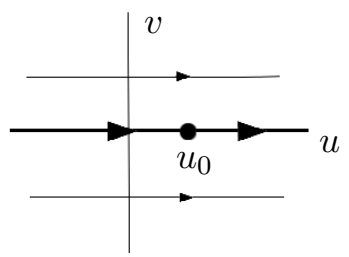

FiguRE 3. Sectorial decomposition of system $(3)$ at $\left(u_{0}, 0\right)$ when $u_{0} \geq 0, p\left(u_{0}\right)=p^{\prime}\left(u_{0}\right)=0$ and $p^{\prime \prime}\left(u_{0}\right)>0$.
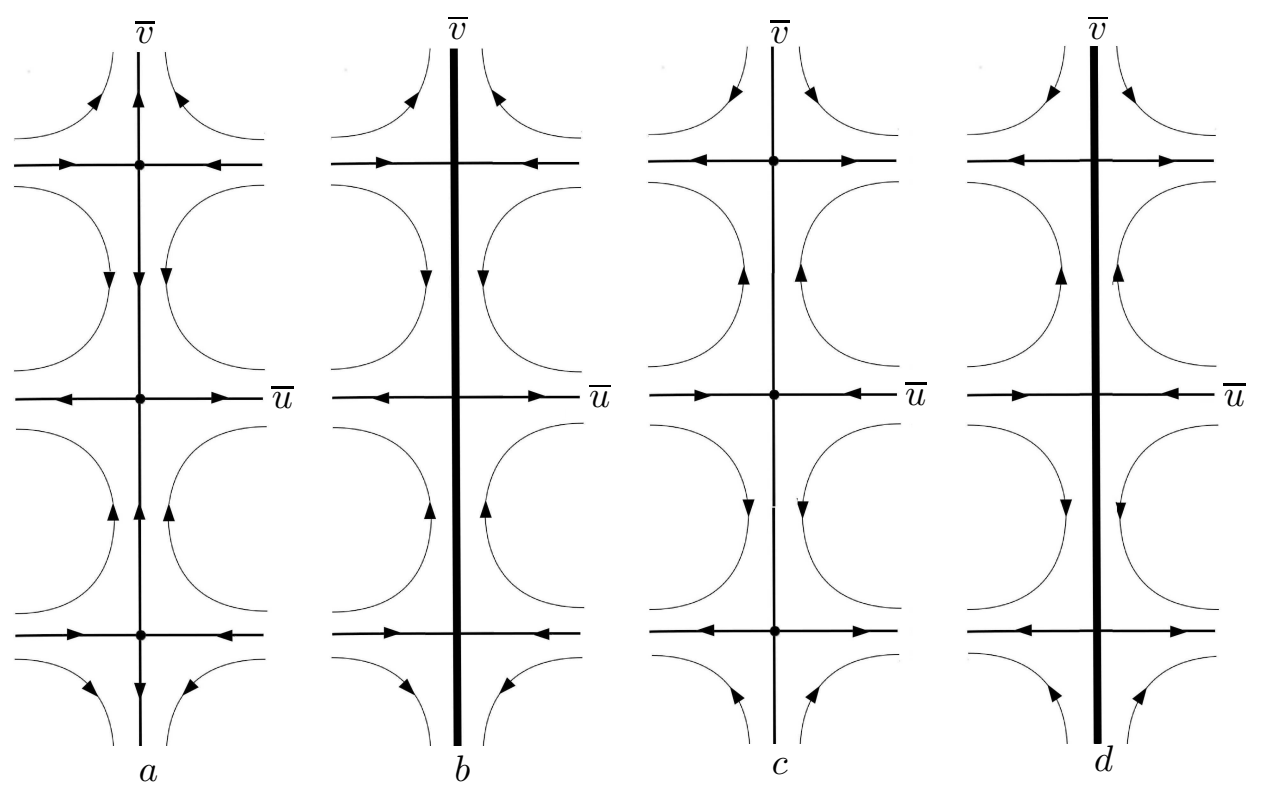

Figure 4. Case $u_{0}=0, p(0)=p^{\prime}(0)=0$ and $p^{\prime \prime}(0)<0$. (a) Blow-up in the negative $u$-direction, system (15), (b) system (14), (c) blow-up in the positive $u$-direction, system (12), (d) system (11).

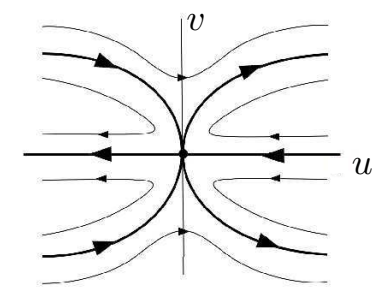

FiguRE 5. Sectorial decomposition of system (3) at the origin when $u_{0}=0, p(0)=p^{\prime}(0)=0$ and $p^{\prime \prime}(0)<0$. 

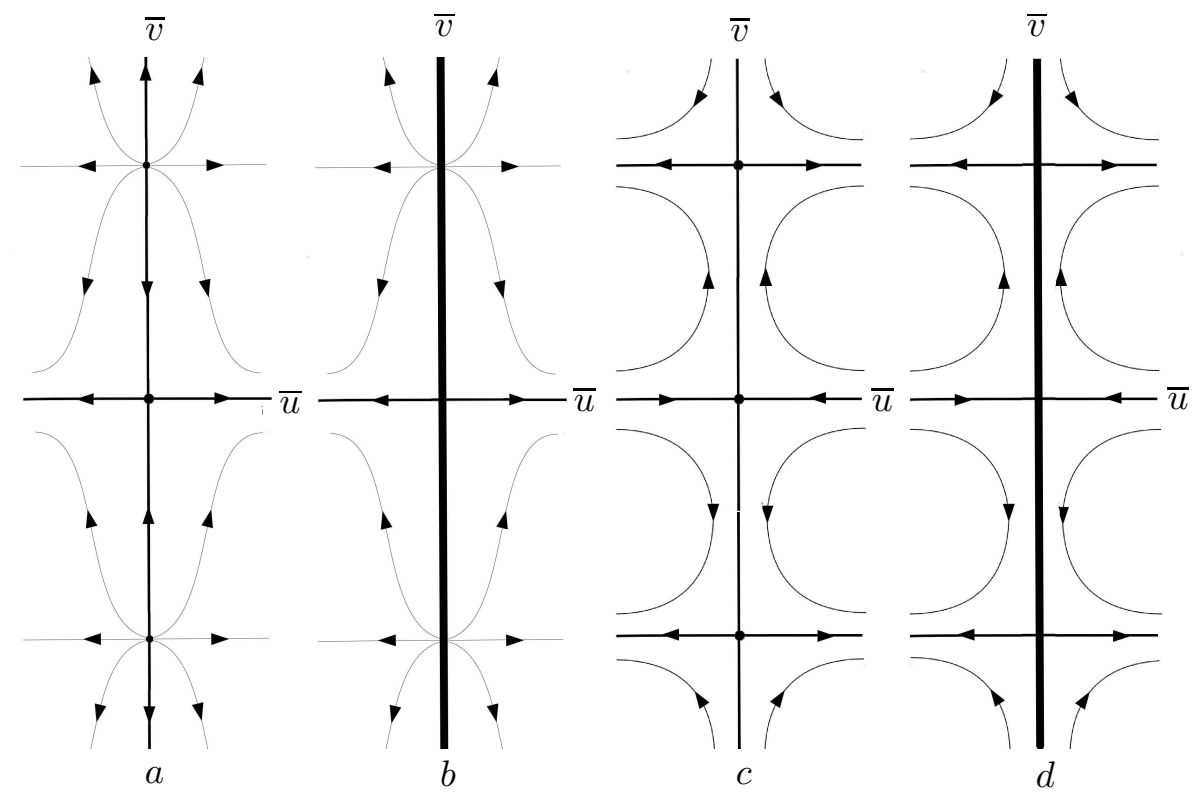

Figure 6. Case $u_{0}>0, p\left(u_{0}\right)=p^{\prime}\left(u_{0}\right)=0$ and $p^{\prime \prime}\left(u_{0}\right)<0$. (a) Blow-up in the negative $u$-direction, system (15), (b) system (14), (c) blow-up in the positive $u$-direction, system (12), (d) system (11).

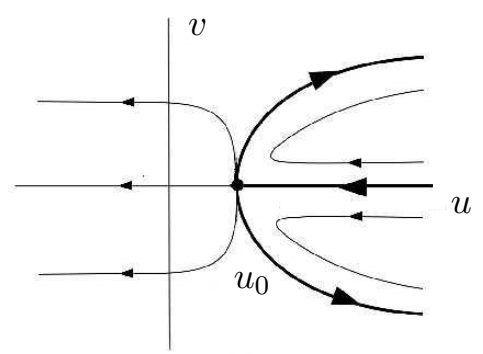

FiguRE 7. Sectorial decomposition of system $(3)$ at $\left(u_{0}, 0\right)$ when $u_{0}>0, p\left(u_{0}\right)=p^{\prime}\left(u_{0}\right)=0$ and $p^{\prime \prime}\left(u_{0}\right)<0$.

and

$$
v=0 \Rightarrow \dot{u}=c u^{4}
$$

For the case $a=b=0$ and $c>0$ we take the blow up $u=\bar{u}, v=\bar{u} \bar{v}$, which carries system (3) into

$$
\begin{aligned}
& \dot{\bar{u}}=\left(1+\bar{u}^{2}\right) \bar{u}^{4} \bar{v}^{4}+c \bar{u}^{4}, \\
& \dot{\bar{v}}=\bar{u}^{5} \bar{v}^{5}-\bar{u}^{3} \bar{v}^{5}\left(1+\bar{u}^{2}\right)-c \bar{u}^{3} \bar{v} .
\end{aligned}
$$


Doing the rescaling of the independent variable given by $d t=\bar{u}^{3} d s$, system (18) becomes

$$
\begin{aligned}
\bar{u}^{\prime} & =\left(1+\bar{u}^{2}\right) \overline{u v}^{4}+c \bar{u}, \\
\bar{v}^{\prime} & =\bar{u}^{2} \bar{v}^{5}-\bar{v}^{5}\left(1+\bar{u}^{2}\right)-c \bar{v} .
\end{aligned}
$$

Then the origin is a hyperbolic saddle point of system (19).

If $c>0$ then we presented the blow-up in Figures 8-a,b. From (16) it follows that the sectorial decomposition of system (3) at the origin is given by Figure 8-c.

For the case $a=b=0$ and $c<0$ we take the blow up $u=\bar{u} \bar{v}, v=\bar{v}$, which carries system (3) into

$$
\begin{aligned}
& \dot{\bar{u}}=\left(1+c \bar{u}^{4}\right) \bar{v}^{3}, \\
& \dot{\bar{v}}=\bar{u} \bar{v}^{6} .
\end{aligned}
$$

Doing the rescaling of the independent variable given by $d t=\bar{v}^{3} d s$, system (20) becomes

$$
\begin{aligned}
& \bar{u}^{\prime}=1+c \bar{u}^{4}, \\
& \bar{v}^{\prime}=\bar{u} \bar{v}^{3} .
\end{aligned}
$$

Then the singularities with $\bar{v}=0$ are $( \pm 1 / \sqrt[4]{-c}, 0)$, which are semi-hyperbolic saddle points.

If $c<0$ then we presented the blow-up in Figures 9-a,b. From (16) and (17) it follows that the sectorial decomposition of system (3) at the origin is given by Figure 10 .
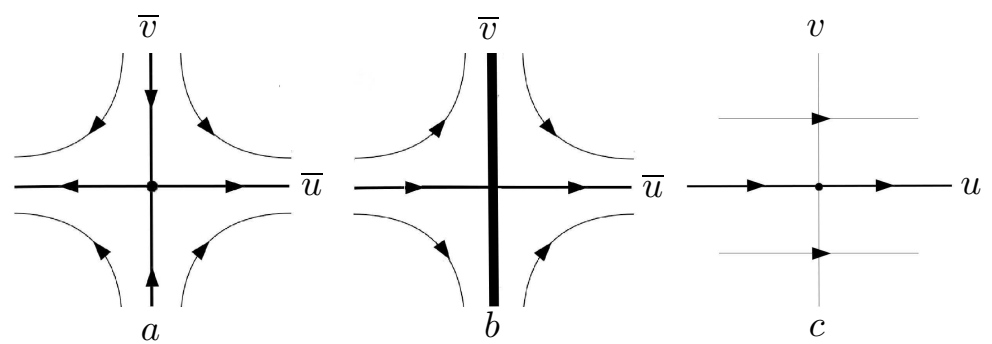

Figure 8. Case $a=b=0$ and $c>0$. (a) Blow-up, system (19), (b) system (18), (c) sectorial decomposition of system (3) at the origin.

6.2. The origin of $U_{2}$. In this section we study the local phase portrait of the origin of $U_{2}$.

Remark 10. For system (4) we have that $\dot{u}<0$ when $u=0, v \neq 0$. Moreover, this system is invariant under the change of coordinates $(t, u, v) \mapsto$ $(-t,-u, v)$. 

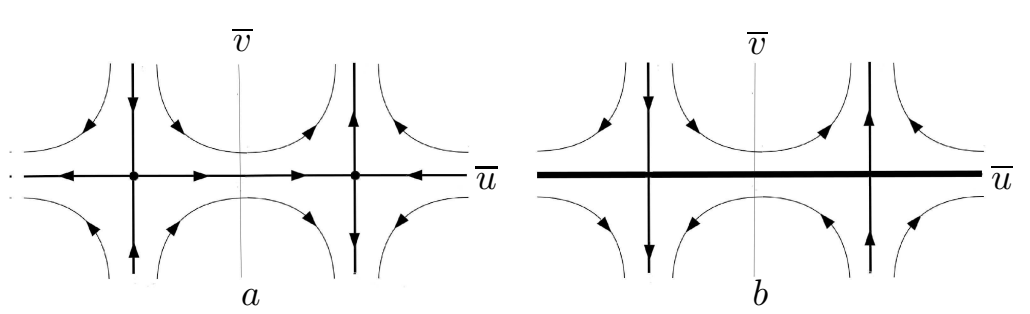

Figure 9. Case $a=b=0$ and $c<0$. (a) Blow-up, system (21), (b) system (20).

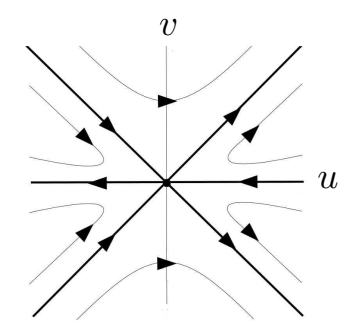

Figure 10. Case $a=b=0, c>0$. Sectorial decomposition of system (3) at the origin.

As in the previous section the choice of the exponents $\alpha$ and $\beta$ of the quasihomogeneous blow-up depends of the order of the vanishing of $q$ at the origin. Then we will divide the study of the origin of $U_{2}$ in the cases $c \neq 0 ; c=0, b \neq 0$ and $c=b=0, a \neq 0$ which will treated in Lemmas 11-13, respectively.

In the next section we will prove that all the possibilities for the sectorial decomposition of system (4) at the origin presented in Lemmas 11 and 12, are realized for an appropriate choice of the parameters $a, b$ and $c$. On the other hand, for the case $a<0$ and $b=c=0$, only the second possibility for the sectorial decomposition of system (4) at the origin presented by Lemma 13 is realized.

Lemma 11. If $c<0$ then there are two possibilities for the sectorial decomposition of system (4) at the origin: either two elliptic sectors, or two elliptic sectors, one parabolic repelling sector and one parabolic attracting sector (Figure 11-c). If $c>0$ then the sectorial decomposition of system (4) at the origin is composed by two hyperbolic sectors, one parabolic repelling sector and one parabolic attracting sector (Figure 12-c).

Proof. Suppose $c \neq 0$. After the blow-up in the positive $u$-direction defined by $u=\bar{u}^{2}, v=\bar{u} \bar{v}$, system (4) becomes

$$
\begin{aligned}
& \dot{\bar{u}}=-\frac{1}{2} \bar{u}^{3}\left[\left(1+\bar{u}^{4}\right) \bar{v}^{4}+q\left(\bar{u}^{2}\right)\right], \\
& \dot{\bar{v}}=\frac{1}{2} \bar{u}^{2} \bar{v}\left[\left(1+\bar{u}^{4}\right) \bar{v}^{4}-q\left(\bar{u}^{2}\right)\right] .
\end{aligned}
$$


Doing the rescaling of the independent variable given by $d t=\bar{u}^{2} d s$, system (22) writes

$$
\begin{aligned}
& \bar{u}^{\prime}=-\frac{1}{2} \bar{u}\left[\left(1+\bar{u}^{4}\right) \bar{v}^{4}+q\left(\bar{u}^{2}\right)\right], \\
& \bar{v}^{\prime}=\frac{1}{2} \bar{v}\left[\left(1+\bar{u}^{4}\right) \bar{v}^{4}-q\left(\bar{u}^{2}\right)\right] .
\end{aligned}
$$

The singular points of system (23) with $\bar{u}=0$ are of the form $\left(0, \bar{v}_{0}\right)$ where $\bar{v}_{0}=0$ or $\bar{v}_{0}$ is a solution of the equation $\bar{v}_{0}^{4}=c$, then $\left(0, \bar{v}_{0}\right)$ is an isolated singular point.

The Jacobian matrix of the vector field defined by system (23) evaluated at $\left(0, \bar{v}_{0}\right)$ is

$$
\left(\begin{array}{cc}
-\frac{1}{2}\left(\bar{v}_{0}^{4}+c\right) & 0 \\
0 & \frac{1}{2}\left(5 \bar{v}_{0}^{4}-c\right)
\end{array}\right)
$$

If $c<0$ then the origin is the unique singular point of (23) with $\bar{u}=0$, moreover, this is a hyperbolic unstable node. The blow-up in the positive $u$-direction is presented in Figures 11-a,b. From Remark 10 it follows that the sectorial decomposition of system (4) at the origin is given by Figure 11-c.

If $c>0$ then there are three singular points of (23) with $\bar{u}=0$, namely: $(0,0)$ is a hyperbolic stable node and $(0, \pm \sqrt[4]{c})$ are hyperbolic saddle points. The blow-up in the positive $u$-direction is presented in Figures 12-a,b. From Remark 10 it follows that the sectorial decomposition of system (4) at the origin is given by Figure 12-c.

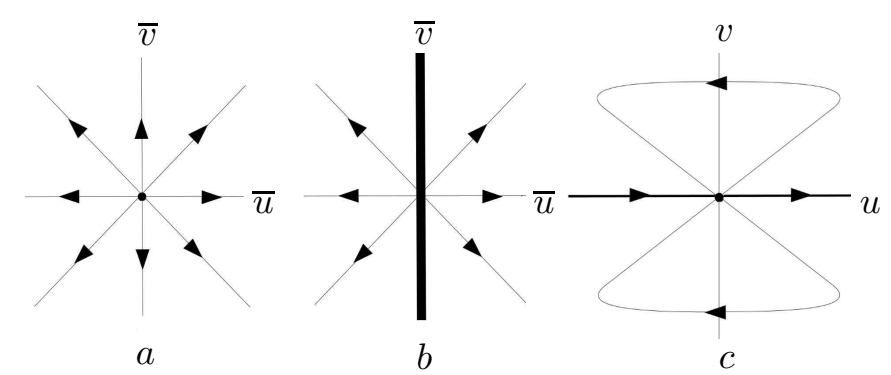

Figure 11. Case $c<0$. (a) Blow-up in the positive $u$-direction, system (23), (b) system (22), (c) sectorial decomposition of system (4) at the origin.

Lemma 12. If $c=0$ and $b<0$ then there are two possibilities for the sectorial decomposition of system (4) at the origin: either two elliptic sectors, or two elliptic sectors, one parabolic repelling sector and one parabolic attracting sector (Figure 13-c). If $c=0$ and $b>0$ then the sectorial decomposition of system (4) at the origin is composed by two hyperbolic sectors (Figure 14-c). 

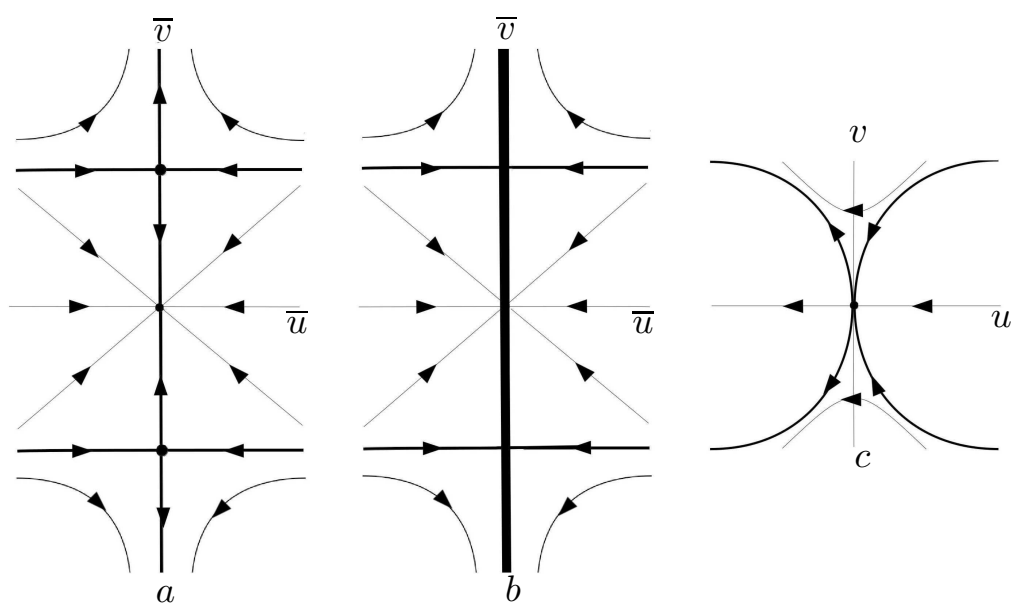

Figure 12. Case $c>0$. (a) Blow-up in the positive $u$-direction, system (23), (b) system (22), (c) sectorial decomposition of system (4) at the origin.

Proof. Suppose that $c=0, b \neq 0$. We take the blow-up defined by $u=\bar{u}, v=$ $\bar{u} \bar{v}$ which carries system (4) into

$$
\begin{aligned}
& \dot{\bar{u}}=-\left(1+\bar{u}^{2}\right) \bar{u}^{4} \bar{v}^{4}-\bar{u}^{4}\left(a \bar{u}^{2}+b\right), \\
& \dot{\bar{v}}=\bar{u}^{3} \bar{v}^{5} .
\end{aligned}
$$

Doing the rescaling of the independent variable given by $d t=\bar{u}^{3} d s$, system (24) becomes

$$
\begin{aligned}
& \bar{u}^{\prime}=-\left(1+\bar{u}^{2}\right) \overline{u v}^{4}-\bar{u}\left(a \bar{u}^{2}+b\right), \\
& \bar{v}^{\prime}=\bar{v}^{5} .
\end{aligned}
$$

The singular point with $\bar{u}=0$ is $(0,0)$ and this is an isolated singularity. The Jacobian matrix of the vector-field defined by system (25) evaluated at $(0,0)$ is

$$
\left(\begin{array}{cc}
-b & 0 \\
0 & 0
\end{array}\right)
$$

If $b<0$ then $(0,0)$ is a semi-hyperbolic unstable node of system $(25)$. The blow-up is presented in Figures 13-a,b. From Remark 10 it follows that the sectorial decomposition of system (4) at the origin is given by Figure 13-c.

If $b>0$ then $(0,0)$ is a semi-hyperbolic saddle point of system $(25)$. The blow-up is presented in Figures 14-a,b. Using Remark 10 it follows that the sectorial decomposition of system (4) at the origin is given by Figure 14-c.

Lemma 13. If $a<0$ and $b=c=0$ then there are two possibilities to the sectorial decomposition of system (4) at the origin: either two elliptic sectors, or two elliptic sectors, one parabolic repelling sector and one parabolic 

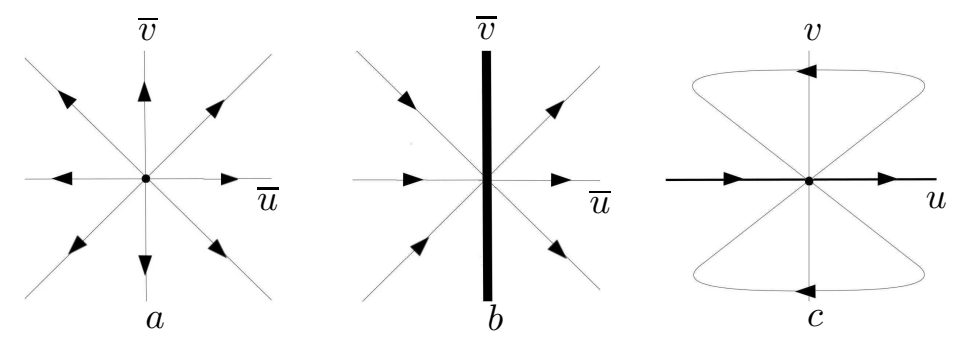

Figure 13. Case $c=0$ and $b<0$. (a) Blow-up, system (25), (b) system (24), (c) sectorial decomposition of system (4) at the origin.
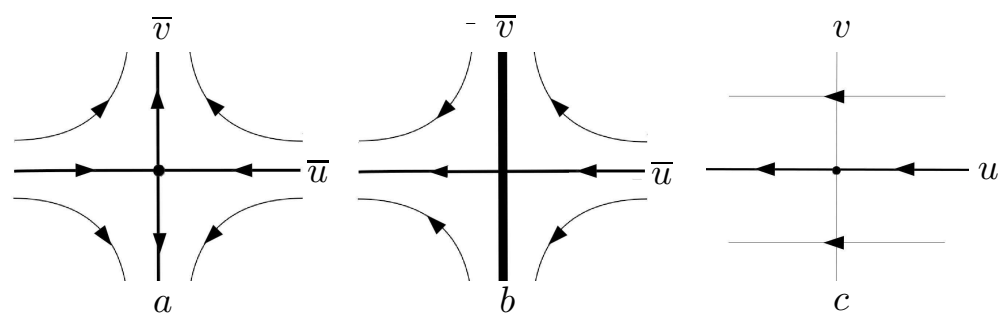

Figure 14. Case $c=0$ and $b>0$. (a) Blow-up, system (25), (b) system (24), (c) sectorial decomposition of system (4) at the origin.

attracting sector (Figure 15-c). If $a>0$ and $b=c=0$ then the sectorial decomposition of system (4) at the origin is composed by two hyperbolic sectors (Figure 16-c).

Proof. Suppose $a \neq 0, b=c=0$. After the blow-up in the positive $u$-direction defined by $u=\bar{u}^{2}, v=\bar{u}^{3} \bar{v}$ system (4) becomes

$$
\begin{aligned}
& \left.\dot{\bar{u}}=-\frac{1}{2} \bar{u}^{11}\left[\left(1+\bar{u}^{4}\right) \bar{v}^{4}+a\right)\right], \\
& \left.\dot{\bar{v}}=-\bar{u}^{10} \bar{v}\left(\bar{u}^{4} \bar{v}^{4}+a\right)+\frac{3}{2} \bar{u}^{10} \bar{v}\left[\left(1+\bar{u}^{4}\right) \bar{v}^{4}+a\right)\right] .
\end{aligned}
$$

Doing the rescaling of the independent variable given by $d t=\bar{u}^{10} d s$, system (26) becomes

$$
\begin{aligned}
& \left.\bar{u}^{\prime}=-\frac{1}{2} \bar{u}\left[\left(1+\bar{u}^{4}\right) \bar{v}^{4}+a\right)\right], \\
& \bar{v}^{\prime}=\frac{1}{2} \bar{v}\left(\bar{u}^{4} \bar{v}^{4}+3 \bar{v}^{4}+a\right) .
\end{aligned}
$$

The singular points of system (27) with $\bar{u}=0$ are isolated and given by $\left(0, \bar{v}_{0}\right)$, where $\bar{v}_{0}=0$ or $\bar{v}_{0}$ is a solution of the equation $3 \bar{v}_{0}^{4}=-a$. The Jacobian matrix of the vector-field defined by $(27)$ evaluated at $\left(0, \bar{v}_{0}\right)$ is

$$
\left(\begin{array}{cc}
-\frac{1}{2}\left(\bar{v}^{4}+a\right) & 0 \\
0 & \frac{1}{2}\left(15 \bar{v}^{4}+a\right)
\end{array}\right) .
$$

If $a<0$ then there are three singular points of system (27) with $\bar{u}=0$, moreover $(0,0)$ is a hyperbolic saddle point, and $(0, \pm \sqrt[4]{-a / 3})$, are hyperbolic unstable nodes. The blow-up in the positive $u$-direction is given by 

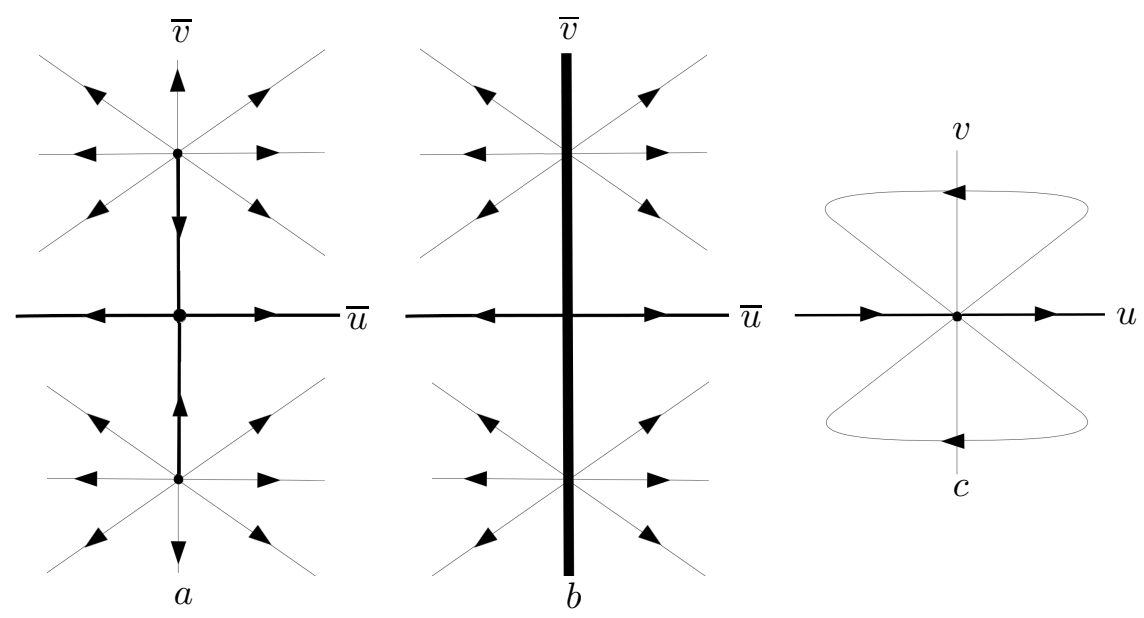

Figure 15. Case $a<0$ and $b=c=0$. (a) Blow-up in the positive $u$-direction, system (27), (b) system (26), (c) sectorial decomposition of system (4) at the origin.
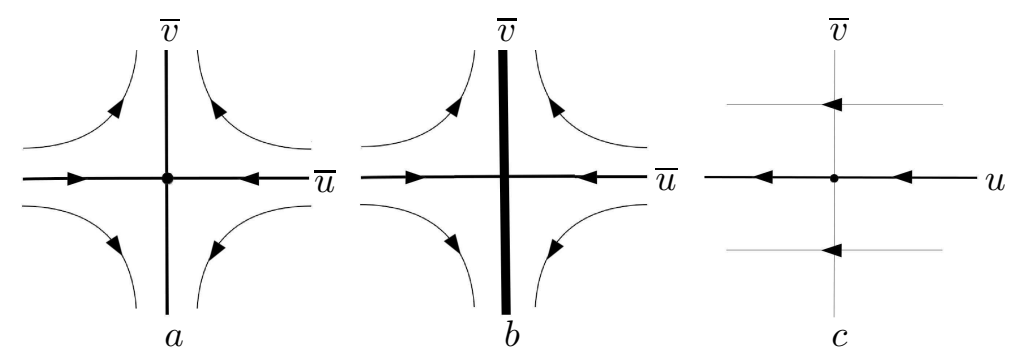

Figure 16. Case $a>0$ and $c=b=0$. (a) Blow-up in the positive $u$ - direction, system (27), (b) system (26), (c) sectorial decomposition of system (4) at the origin.

Figures 15-a,b. From Remark 10 it follows that the sectorial decomposition of system (4) at the origin is given by Figure 15-c.

If $a>0$ then the origin is the unique singular point of system (27) with $\bar{u}=0$, and it is a hyperbolic saddle point. We presented the blow-up in the positive $u$-direction in Figures 16-a,b. From Remark 10 it follows that the sectorial decomposition of system (4) at the origin is given by Figure 16 -c.

\section{Proof of Theorem 1}

The proof of Theorem 1 is a combination of Lemmas 14, 15 and Remark 16. In Lemma 14 we use a linear change of coordinates to find normal forms of system (2). Finally, in Lemma 15, we determine the possibilities for the global phase portrait in the Poincaré disk of each normal form. 


\begin{tabular}{||l|l||}
\multicolumn{1}{|c|}{ System (2) } & \multicolumn{1}{c||}{ Normal form (i)-(viii) } \\
\hline \hline$a=b=c=0$ & (i) \\
\hline$a=b=0, c \neq 0$ & (ii), $A=c /|c|$ \\
\hline$a=0, b \neq 0, c=0$ & (iii), $A=b /|b|$ \\
\hline$a=0, b \neq 0, c \neq 0$ & (iv), $A=b /|b|, C=c /|b|$ \\
\hline$a \neq 0, b=c=0$ & (v), $A=a /|a|$ \\
\hline$a \neq 0, b=0, c \neq 0$ & (vi), $A=a /|a|, C=c /|a|$ \\
\hline$a \neq 0, b \neq 0, c=0$ & (vii), $A=a /|a|, B=b /|a|$ \\
\hline$a \neq 0, b \neq 0, c \neq 0$ & (viii) $A=a /|a|, B=b /|a|, C=c /|a|$ \\
\hline
\end{tabular}

TABLE 2. Correspondence between the parameters of system (2) and the parameters of normal forms.

Lemma 14. All systems of the form (2) are topologically equivalent to one of the normal forms (i)-(viii) for an appropriate choice of $A \in\{-1,1\}, B, C \in$ $\mathbb{R} \backslash\{0\}$. The correspondence between parameters of system (2) and parameters of its respective normal form is given in Table 2.

Proof. Fixed $\delta \neq 0$, after the linear change of coordinates $(x, y) \mapsto(\delta x, \delta y)$ system (2) becomes

$$
\dot{x}=-y, \dot{y}=x+\frac{a}{\delta^{4}} x^{5}+\frac{b}{\delta^{4}} x^{3} y^{2}+\frac{c}{\delta^{4}} x y^{4} .
$$

Considering appropriate choice of $\delta=\sqrt[4]{ \pm a}, \delta=\sqrt[4]{ \pm b}$ or $\delta=\sqrt[4]{ \pm c}$ we obtain Table 2.

Lemma 15. The phase portrait in the Poincaré disk of each normal form (i)-(viii) is topologically equivalent to one of the phase portraits of Figure 1. The possibilities for the global phase portrait in the Poincaré disk for each normal form (i)-(viii) are presented in the first and third columns of Table 1 .

Proof. From [5] it follows that the origin is a center of all normal form (i)-(viii). Combining informations of Remarks 4, 5, Lemmas 6-13 and the symmetries of system (2), we obtain the local phase portraits in the Poincaré disk presented in Figure 17. The correspondence between normal forms and local phase portraits of Figure 17 is given in Table 3.

Since system (2) is linear when $a=b=c=0$, the global phase portrait in the Poincaré disk of the vector field corresponding to Figure 17-a is given by Figure 1-a.

Now we will determine the global phase portrait in the Poincaré disk of the normal forms corresponding to Figure 17-b. In this case, the finite singularities of $X$ are the origin, which is a center, and two hyperbolic saddle points, see Remark 4. Notice that from the definition of system (2) it follows 

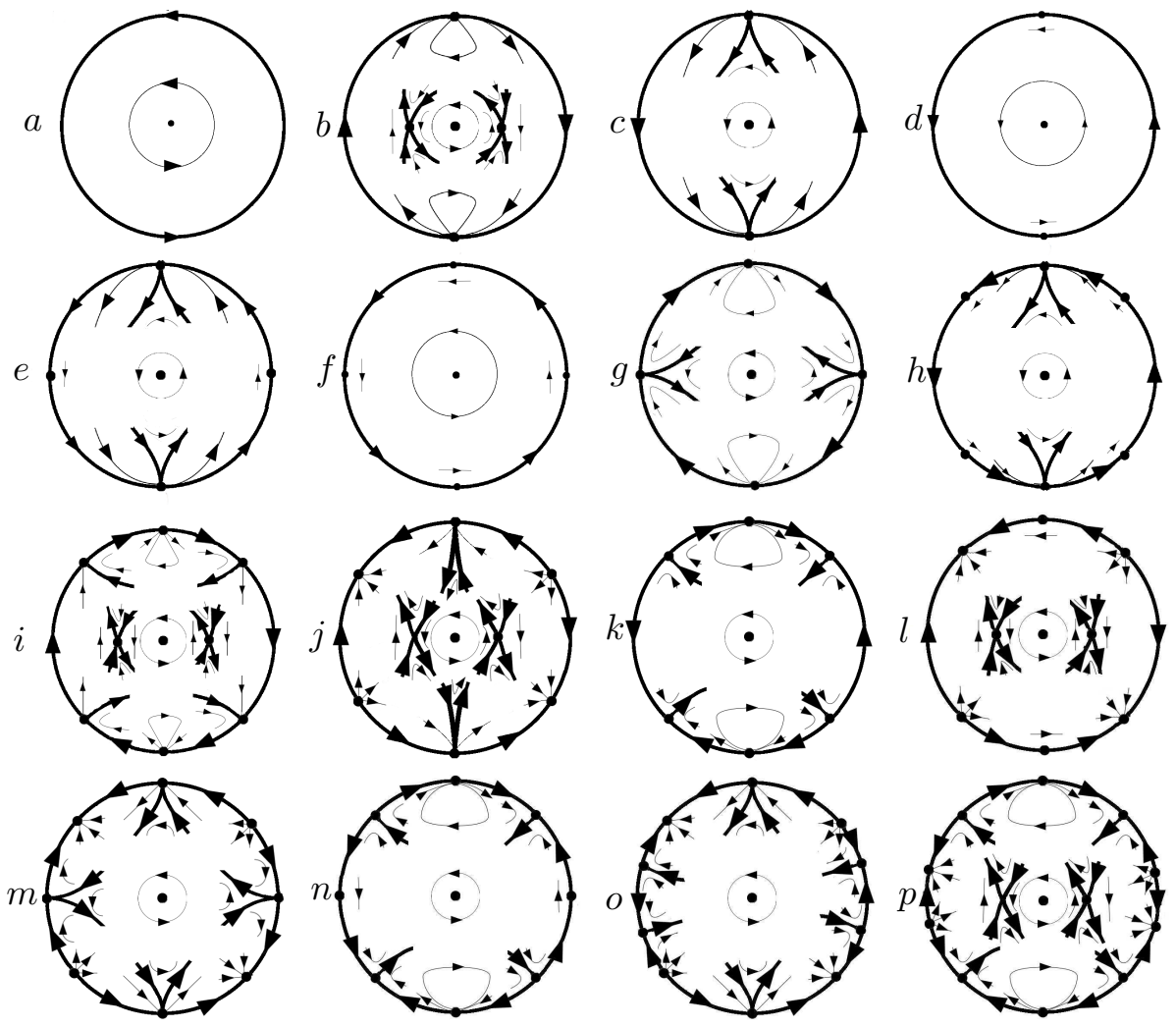

FiguRE 17. Local phase portraits in the Poincaré disk of normal forms (i)-(viii).

that $\dot{x}<0$ and $\dot{y}=0$ if $x=0$ and $y>0 ; \dot{x}>0$ and $\dot{y}=0$ if $x=0$ and $y<0$. Moreover, $\dot{x}=0$ and $\dot{y}>0$ when $x \in(-\infty,-1) \cup(0,1)$ and $y=0 ; \dot{x}=0$ and $\dot{y}<0$ when $x \in(-1,0) \cup(1,+\infty)$ and $y=0$. Combining these informations with the symmetries of system (2), we obtain that the global phase portrait in the Poincaré disk of the normal forms corresponding Figure 17-b is given by Figure 1-b.

In the same way we can prove that the global phase portrait in the Poincaré disk of normal forms corresponding to Figure 17-a-i is given by Figure 1-a-i, respectively. Also, the global phase portrait in the Poincaré disk of normal forms corresponding to Figure 17-k,l,n is given by Figure 1-m,n,r, respectively.

With the same arguments we can prove that for each normal form corresponding to the Figure $17-\mathrm{j}, \mathrm{m}$, o there exist three possibilities for the global phase portrait in the Poincaré disk, which are presented in Figures 1-j,k,l, Figures 1-o,p,q and Figures 1-s,t,u, respectively.

Finally, we will prove that the global phase portrait in the Poincare disk of normal form corresponding Figure 17-p is given by Figure 1-v. In fact, 


\begin{tabular}{|c|c|}
\hline $\begin{array}{l}\text { Local Phase } \\
\text { Portrait of } \\
\text { Figure } 17 \\
\end{array}$ & Normal Form \\
\hline $\mathrm{a}$ & (i) \\
\hline b & $\begin{array}{l}\text { (v), } A=-1 \\
\text { (viii), } A=-1, B>0, C<0, B^{2}+4 C<0 \\
\text { (viii), } A=-1, B<0, C<0 \\
\text { (vii), } A=-1, B<0 \\
\text { (vi), } A=-1, C<0\end{array}$ \\
\hline $\mathrm{c}$ & $\begin{array}{l}\text { (viii), } A=1, B<0, C>0, B^{2}-4 C<0 \\
\text { (viii) } A=1, B>0, C>0 \\
\text { (vi), } A=1, C>0\end{array}$ \\
\hline $\mathrm{d}$ & $\begin{array}{l}\text { (v), } A=1 \\
\text { (vii), } A=1, B>0\end{array}$ \\
\hline $\mathrm{e}$ & $\begin{array}{l}\text { (ii), } A=1 \\
\text { (iv), } A=1, C>0\end{array}$ \\
\hline $\mathrm{f}$ & (iii), $A=1$ \\
\hline $\mathrm{g}$ & $\begin{array}{l}\text { (ii), } A=-1 \\
\text { (iii), } A=-1 \\
\text { (iv), } A=-1, C<0\end{array}$ \\
\hline $\mathrm{h}$ & (viii), $A=1, B<0, C>0, B^{2}-4 C=0$ \\
\hline i & (viii), $A=-1, B>0, C<0, B^{2}+4 C=0$ \\
\hline $\mathrm{j}$ & $\begin{array}{l}\text { (viii), } A=-1, B>0, C>0 \\
\text { (viii), } A=-1, B<0, C>0 \\
\text { (vi), } A=-1, C>0\end{array}$ \\
\hline $\mathrm{k}$ & $\begin{array}{l}\text { (viii), } A=1, B \neq 0, C<0 \\
\text { (vii), } A=1, B<0 \\
\text { (vi), } A=1, C<0\end{array}$ \\
\hline 1 & (vii), $A=-1, B>0$ \\
\hline $\mathrm{m}$ & (iv), $A=-1, C>0$ \\
\hline $\mathrm{n}$ & (iv), $A=1, C<0$ \\
\hline $\mathrm{O}$ & (viii), $A=1, B<0, C>0, B^{2}-4 C>0$ \\
\hline $\mathrm{p}$ & (viii), $A=-1, B>0, C<0, B^{2}+4 C>0$ \\
\hline
\end{tabular}

TABLE 3. Classification of local phase portraits in the Poincaré disk of normal forms (i)-(viii).

first observe that, in this case, for the correspondent system (3) in $\mathbb{R}^{2}$, the union of the points $(u, v)$ such that $\dot{u}=0$ is the union $\Lambda$ of the graphics of the functions $v= \pm \sqrt[4]{-p(u) /\left(1+u^{2}\right)}$; the points $(u, v)$ for which $\dot{u}>0$ are given by region $R^{+}$in Figure 18. The region $R^{-}$corresponds to the points $(u, v)$ such that $\dot{u}<0$. 


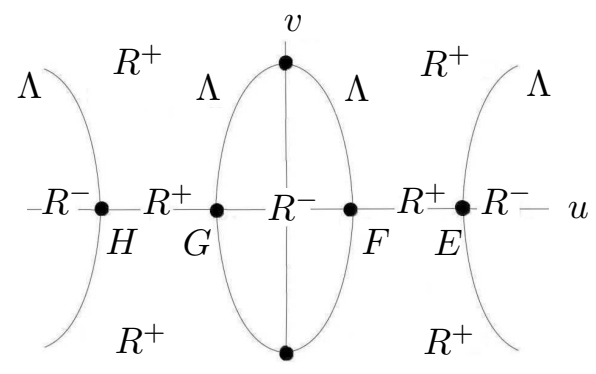

Figure 18. Signs of $\dot{u}$ for system (3) corresponding to case of Figure 17-p.

To complete the proof, it is sufficient to shows that $\alpha(\Gamma)=\{F\}$ in Figure 19-a, where $\alpha(\Gamma)$ denotes the $\alpha$-limit of the orbit $\Gamma$. Suppose that this assertion is false, then $\alpha(\Gamma)=\{D\}$ or $\alpha(C)=\{E\}$. The phase portraits of system $(3)$ in $\mathbb{R}^{2}$, for cases $\alpha(\Gamma)=\{D\}$ and $\alpha(\Gamma)=\{E\}$, are presented in Figures 19-b,c, respectively. If $\alpha(\Gamma)=\{D\}$ then an integral curve which connect points $E$ and $H$ provides a contradiction with the sign of $\dot{u}$ in region $R^{+}$presented in Figure 18. In the same way, if $\alpha(\Gamma)=\{E\}$ then $\Gamma$ contradicts Figure 18.
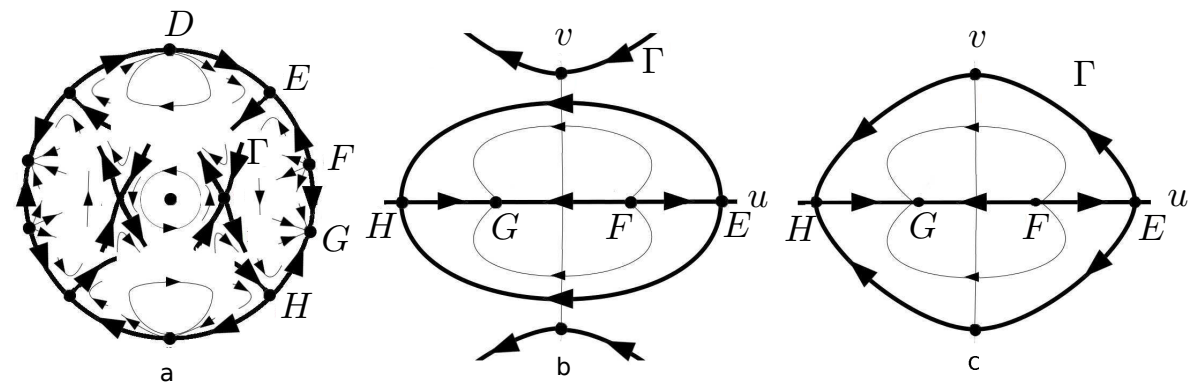

FiguRE 19. (a) Local phase portrait in the Poincaré disk (b) global phase portrait in $\mathbb{R}^{2}$ of system $(3)$, if $\alpha(C)=\{A\}$, (c) global phase portrait in $\mathbb{R}^{2}$ of system $(3)$, if $\alpha(C)=\{B\}$.

Remark 16. Using Program $P_{4}$ we can check that the global phase portraits in the Poincaré disk of Figures $1-\mathrm{j}, 1$, are realized by choosing $a=-1, b \in$ $\{-1,0,1\}, c=20$ and $a=-1, b \in\{-1,0,1\}, c=1$, in system (2), respectively. Then, by continuity, choosing $a=-1$ and $b \in\{-1,0,1\}$, the global phase portrait in the Poincaré disk of Figure 1-k is realized for an appropriate choice of $c \in(1,20)$. In the same way, the global phase portraits in the Poincaré disk of Figures 1-o,q are realized by the choice $a=0, b=-1, c=10$ and $a=0, b=-1, c=0.1$, respectively. By continuity, the global phase portrait in the Poincaré disk of Figure 1-p is realized for $a=0, b=-1$ and an 
appropriate choice of $c \in(0.1,10)$. Finally, the global phase portraits in the Poincaré disk of Figures 1-s, $\mathrm{u}$, are realized choosing $a=1, b=-2.1, c=1$ and $a=1, b=-10, c=1$, respectively. By continuity, the global phase portrait in the Poincaré disk of Figure 1-t is realized for $a=-1, c=1$ and an appropriate choice of $c \in(-10,-2.1)$.

\section{REFERENCES}

[1] C.A. Buzzi, J. Llibre, J.C. Medrado, Phase portraits of reversible linear differential systems with cubic homogeneous polynomial nonlinearities having a non-degenerate center at the origin, Qual. Theory Dyn. Syst. 7 (2009), 369-403.

[2] R. Benterki and J. Llibre, Centers and their perturbation for the Kukles homogeneous polynomial differential systems of degree 4 symmetric with respect to the $y$-axis, preprint (2015).

[3] F. Dumortier, J. Llibre And J.C. Artés, Qualitative Theory of Planar Differential Systems, Springer, 2006.

[4] J. Giné, Conditions for the existence of a center for the Kukles homogeneous systems, Comput. Math. Appl. 43 (2002), 1261-1269.

[5] J. Giné, J. Llibre And C. Valls, Centers for the Kukles homogeneous systems with odd degree, to appear in Bull. London Math. Soc. (2015), doi 10.1112/blms/bdv005.

[6] J. Giné, J. Llibre and C. VAlls, Centers for the Kukles homogeneous systems with even degree, preprint (2015).

[7] J. Llibre AND T. SAlHI, Centers and their perturbation for the Kukles homogeneous polynomial differential systems of degree 4 symmetric with respect to the $x$-axis, preprint (2015).

[8] K.E. MALKIN, Criteria for the center for a certain differential equation, (Russian) Volz. Mat. Sb. Vyp. 2 (1964), 87-91.

[9] L. Markus, Global structure of ordinary differential equations in the plane, Trans. Amer. Math. Soc. 76 (1954), 127-148.

[10] D. Neumann, Classification of continuous flows on 2-manifold, Proc. Amer. Math. Soc. 48 (1975), 73-81.

[11] E. P. Volokitin, V. V. Ivanov, Isochronicity and commutation of polynomial vector fields, Siberian Math. J. 40 (1999), 22-37.

[12] N.I. VulPe AND K.S. SibiRskiI, Centro-affine invariant conditions for the existence of a center of a differential system with cubic nonlinearities, (Russian) Dokl. Akad. Nauk SSSR 301 (1988), 1297-1301; translation in Soviet Math. Dokl. 38 (1989), 198201.

[13] H. ŻoŁA̧DEK, The classification of reversible cubic systems with center, Topol. Methods Nonlinear Anal. 4 (1994), 79-136.

[14] H. Żo€A̧DEK, Remarks on: "The classification of reversible cubic systems with center" [Topol. Methods Nonlinear Anal. 4 (1994), 79-136; Topol. Methods Nonlinear Anal. 8 (1996), 335-342.

${ }^{1}$ Departament de Matemàtiques, Universitat Autònoma de Barcelona, 08193

Bellaterra, Barcelona, Catalonia, Spain

E-mail address: jllibre@mat.uab.cat

2 Departamento de Matemática, Universidade Federal de Santa Maria, RS, BRAZIL

E-mail address: mauriciofronzadasilva@hotmail.com 